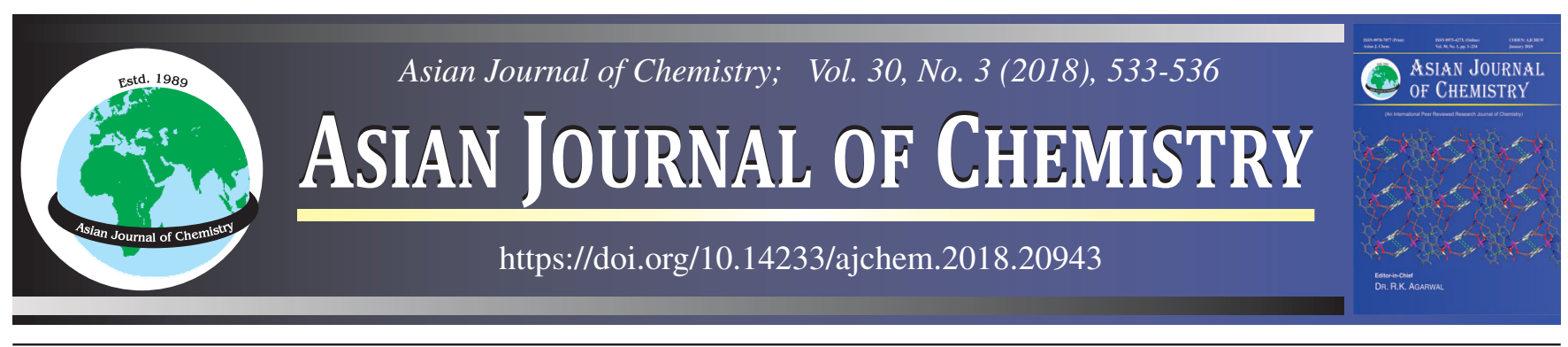

\title{
Effect of Acetone Extract of Sesame (Sesamum indicum L.) and Sunflower (Helianthus annus L.) Meal on Stability of Crude and Refined Soybean Oil
}

\author{
AnJAni ${ }^{*}$, Mukhan Wati and M. KhabiRuddin
}

Department of Chemistry and Biochemistry, Chaudhary Charan Singh Haryana Agricultural University, Hisar-125 004, India

*Corresponding author: E-mail: anjali.roshni5@gmail.com

The antioxidative effect of acetone extracts of sesame and sunflower meal on stability of crude and refined soybean oil (C\&RSO) stored for 120 days at $50{ }^{\circ} \mathrm{C}$ was determined by monitoring the free fatty acid, $p$-anisidine value and peroxide value. The oil was dosed with different concentrations $(500,1000$ and $2000 \mathrm{ppm})$ of the extracts and tert-butylated hydroxyquinone and propyl gallate (conc. of both $200 \mathrm{ppm})$. Free fatty acid, $p$-anisidine value and peroxide value of crude and refined soybean oil containing sesame and sunflower meal extract were lower than crude and refined soybean oil containing no additive. However, refined soybean oil had much higher free fatty acid, $p$-anisidine value and peroxide value than crude soybean oil because some natural antioxidants such as carotene, tocopherol lost during refining. The sesame and sunflower extract have better antioxidant activity than propyl gallate but less than tert-butylated hydroxyquinone. Result suggest that sesame and sunflower meal extract could be used as alternative natural antioxidants with wide food applications.

Keywords: Sesame, Sunflower, tert-Butylated hydroxyquinone, Propyl gallate, Soybean oil.

\section{INTRODUCTION}

One of the major problems of storing crude and refined vegetable oils is oxidation that leads to the development of rancidity and deterioration of the oil quality. Products with higher lipid concentration are naturally considered to be more prone to oxidative rancidity. This happens due to the multiple unsaturated bonds present in the predominant unsaturated fatty acids such as oleic acid, linoleic acid and linolenic acid. Besides affecting the nutritional quality of the products, during this process, it may generate potential toxic compounds through the action of free radicals and reactive oxygen species (ROS) that are harmful for human health and is implicated in degenerative diseases such as cancer and early ageing [1]. The addition of antioxidants to oil is one of the most efficient ways to prevent oxidation reaction between molecular oxygen and unsaturated fatty acids present in oils. Synthetic antioxidants such as butylated hydroxy toluene (BHT), butylated hydroxy anisole (BHA), tert-butylated hydroxy-quinone (TBHQ) and propyl gallate (PG) have been proved effective antioxidants against oil rancidity [2]. tert-Butylated hydroxyquinone is known to be a very effective antioxidant for vegetable oils and it is stable at high temperature [3]. But the use of most of these synthetic antioxidants as additives have been discouraged in international market due to their potential health risks corresponding to carcinogenicity [1,4]. Therefore, more attention has been directed towards bioactive plant extracts (natural antioxidants) which offer a unique range of applications for holistic health and wellness. The antioxidant activity of these extract depend on the isolation procedures and type of active components of extracts [5]. The effectiveness of antioxidants varies depending on the temperature and conditions of processing and storage [3].

Sesame (Sesamum indicum L.) is an important source of edible oil because of its high content of lipid [6]. It is not only good source of edible oil but also widely used in baked goods and confectionery products [7]. The oil shows remarkable stability despite of high unsaturation. Sesame cake is a by-product of sesame oil industry. In some countries, sesame cake is used as poultry feed. Studies show that a significant amount of antioxidant compounds still existed in the sesame meal [8]. Sesame seed meal was studied as potential antioxidant agents due to their high antioxidant activities $[9,10]$. Furthermore, there is a high potential for the commercial isolation of these products due to the low value of sesame seed meals that are being discarded as by-products.

Sunflower (Helianthus annus L.) is the second largest oilseed crop and one of the major protein meals used for livestock feeding and particularly for ruminant diets. Because of its high content of protein, sunflower meal is used primarily in ruminant feed, but its nutritional, sensory and functional properties also make a great interest for human food as a protein source. Sunflower meal is also rich in minerals, valuable vitamins A, E, phenolic acids, polyphenols, flavonoids and condensed tannins and studied as a potential source of cheap natural antioxidants [11]. Sunflower 
meal was studied for phenolic and antioxidant potential due to its high antioxidant activity [12]. Hence, the objectives of present investigation is to prepare extract of sesame and sunflower seed meal, to extract and refine the crude oil of soybean seed obtained from solvent extraction and to study the oxidative stability of crude and refined soybean oil supplemented with sesame and sunflower meal extract against oxidation during accelerated storage in comparison with synthetic antioxidant such as propyl gallate and tert-butylated hydroxyquinone.

\section{EXPERIMENTAL}

The seeds of soybean, sesame meal and sunflower meal were procured from the local market at Hisar, India.

Sample preparation: A commercial sample of sesame and sunflower meals were dried and then ground into a fine powder. Samples (100 g) were defatted with hexane (3 times $\times 500 \mathrm{~mL})$ at room temperature. The defatted residue was washed with distilled water $(3$ times $\times 500 \mathrm{~mL})$ and dried at $50^{\circ} \mathrm{C}$. The above residue $(10 \mathrm{~g})$ was extracted with $150 \mathrm{~mL}$ acetone by Soxhlet method for $8 \mathrm{~h}$. Extracts were filtered and the excess solvent was removed in a rotary evaporator below $40^{\circ} \mathrm{C}$ ) and finally weighed. The residue was redissolved in $100 \mathrm{~mL}$ of acetone to give an antioxidant solution of known concentration and stored in refrigerator for further use.

Extraction of soybean oil: The seeds of soybean were ground to powder. Oil was extracted by Soxhlet method using petroleum ether $\left(60-80{ }^{\circ} \mathrm{C}\right)$ for $8 \mathrm{~h}$.

Refining of soybean oil: Degumming is the first step of refining and involves the removal of easily hydralable phospholipids and metals from the oil. For this, the crude oil was treated with water at room temperature. The hydrated phospholipids are removed at the end of this step.

Step 2: Small amount of phosphoric acid was added to convert the remaining non-hydralable phospholipids $(\mathrm{Ca}, \mathrm{Mg}$ salts) into hydralable phospholipids. Free fatty acids are responsible for oil acidity. During neutralization, the oil was treated with excess $\mathrm{NaOH}$ which reacts with free fatty acid to form soap stock. The soap stock was separated from the oil by centrifugation.

Step 3: The purpose of bleaching (or decolourizing) is to reduce the levels of pigments such as carotenoids and chlorophyll, but this treatment also further removes residues of phosphatides, soaps, traces of metals, oxidation products and proteins. Bleaching was done with acid-activated clay minerals to adsorb colouring components and to decompose hydroperoxides.

Step 4: Deodourization is a vacuum steam distillation process that removes the relatively volatile components, aldehydes and ketones that give rise to undesirable flavours, colours and odours in oil. This is feasible because of the great differences in volatility between these undesirable substances and the oil. The purpose of deodourization is to remove odours, off-flavours and other volatile components such as pesticides and light polycyclic aromatic hydrocarbons. Depending on the residence time in the deodouriser, the process was carried out at low pressure (2-6 mbar) and elevated temperatures $\left(180-270^{\circ} \mathrm{C}\right)$, since the substances responsible for odours and flavours are usually volatile.

Addition of additives to crude and refined soybean oils: Acetone extracts of sesame and sunflower meals at concentrations (200, 500, 1000 and $2000 \mathrm{ppm}$ ) were separately added to crude and refined soybean oils. Experiments were also carried out with synthetic antioxidants propyl gallate and tert-butylated hydroxyquinone at $200 \mathrm{ppm}$, and control set without added antioxidants. Each container was appropriately labelled and samples were stored in uniform glass beaker at $50^{\circ} \mathrm{C}$ for a definite period in an incubator.

Physio-chemical analysis: Samples were analyzed after 20, 40, 60, 80, 100, 120 days to follow the oxidative changes. Required quantity of the sample was taken out periodically and studied for free fatty acids (FFA), $p$-anisidine value (p$\mathrm{AV}$ ) and peroxide value (PV). Free fatty acids and peroxide value were determined according to AOAC method [13]. $p$ Anisidine value was measured according to AOCS method [14]. All the experiments were conducted with duplicate sets and analyses of sample run in triplicate and averaged. The above experiments were repeated with refined soybean oil.

\section{RESULTS AND DISCUSSION}

Effect on peroxide value: Peroxide value is a measure of the concentration of peroxides and hydroperoxides formed in the initial stages of lipid oxidation. The hydroperoxides formed from lipid oxidation are decomposed to volatile carbonyl compounds or non-volatile oxidized dimers, trimers or polymers by further oxidation. For the present investigation, crude and refined soybean oil having an initial peroxide value of 3.4 and $1.1 \mathrm{meq} / \mathrm{Kg}$, respectively was taken. Degree of oxidation of crude and refined soybean oil samples were determined by measuring peroxide value in the absence and presence of antioxidants at $50^{\circ} \mathrm{C}$ for 120 days. The influence of antioxidants during storage on peroxide value in the crude soybean oil samples is shown in Fig. 1. Crude soybean oil (CSO) results showed that peroxide value increased linearly with storage time and increased in acceleration after 60 days. It was observed that maximum increase in peroxide value was observed in control while tertbutylated hydroxyquinone had minimum increase. Crude soybean oil sample without the antioxidant (control) reached a maximum peroxide value of $200.54 \mathrm{meq} / \mathrm{Kg}$ after storage of 120 days. Crude soybean oil containing sesame and sunflower extracts had lower peroxide value values than oil sample containing $200 \mathrm{ppm}$ propyl gallate during storage. As the concentration of the extracts increases, propyl gallate of crude soybean oil gradually decreases. The result observed was in agreement with the observations reported by Nyam et al. [15] for peroxide value of sunflower oil mixed with kenaf seeds extract, roselle seeds extract and roselle extract. Fig. 2 revealed the peroxide value of refined soybean oil (RSO) stored for 120 days. Results of refined soybean oil also showed that peroxide value increased linearly with storage time and increased in acceleration after 40 days. Refined soybean oil sample without the antioxidant (control) reached a maximum peroxide value of 240.24 meq/ $\mathrm{Kg}$ after 120 days of storage. The peroxide value of oil containing antioxidants was lower than peroxide value of oil which contained no additive (control). From the results, it showed that peroxide value of refined oil reached a higher value than crude oil because natural antioxidants such as total tocopherol, sterol and carotenoid had been lost during refining [16]. Peroxide value of the samples having sesame and sunflower extracts were less than propyl gallate but more than tert-butylated 


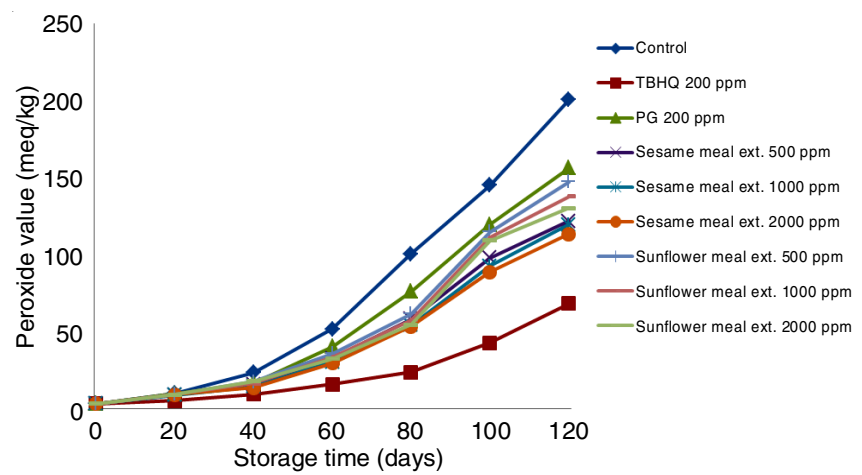

Fig. 1. Comparison of peroxide value (meq/kg) of crude soybean oil stored at $50{ }^{\circ} \mathrm{C}$

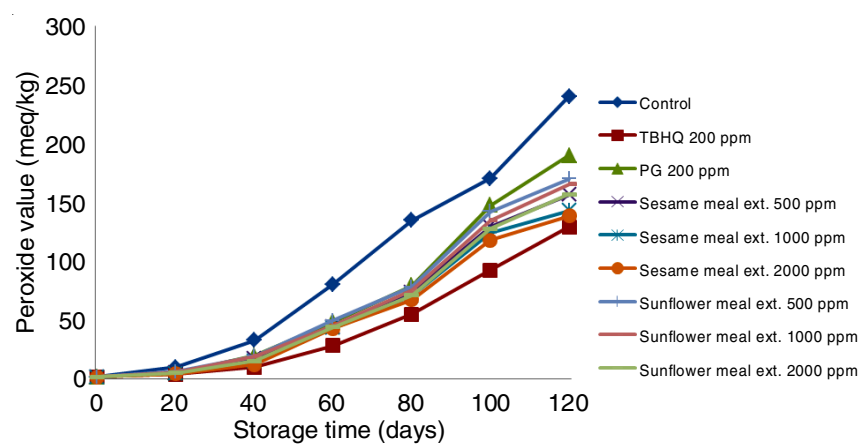

Fig. 2. Comparison of peroxide value (meq $/ \mathrm{kg}$ ) of refined soybean oil stored at $50{ }^{\circ} \mathrm{C}$

hydroxyquinone (TBHQ). Thus sesame and sunflower meal extracts controlled peroxide value appreciably by delaying the hydroperoxides formation and had better stabilization effect than propyl gallate. Although both sesame and sunflower meal extract controlled peroxide value but sesame meal extract is more effective than sunflower meal extract.

Effect on free fatty acid: Free fatty acid is an important oil quality indicator during each stage of processing. Free fatty acid of any lipid is measure of hydrolytic rancidity [17]. Higher value of free fatty acid of any lipid, higher the degree of hydrolytic rancidity that set-in [18]. In crude oil, free fatty acid estimates the amount of oil that will be lost during refining steps designed to remove fatty acids. Fig. 3 depicted the free fatty acid of crude soybean oil stored with natural as well as synthetic antioxidants for 120 days, respectively. Crude soybean oil results showed that free fatty acid increased with storage time and sample without antioxidant (control) reached a maximum of 6.55 (\% oleic acid) after 120 days of storage. Crude soybean oil sample with tert-butylated hydroxyquinone showed minimum increase in free fatty acid during storage. Natural extracts had less stability than tert-butylated hydroxyquinone while more than propyl gallate. The effect of antioxidants during storage on free fatty acid in the refined soybean oil samples is shown in Fig. 4. The free fatty acid of refined soybean oil samples which contained sesame and sunflower extracts, 200 ppm propyl gallate and $200 \mathrm{ppm}$ tert-butylated hydroxyquinone was lower than free fatty acid of oil sample that contained no additive. Free fatty acid of refined soybean oil containing sesame and sunflower extracts was lower than free fatty acid of oil containing $200 \mathrm{ppm}$ propyl gallate. The result is in agreement with Arawande et al. [19]. Free fatty acid of

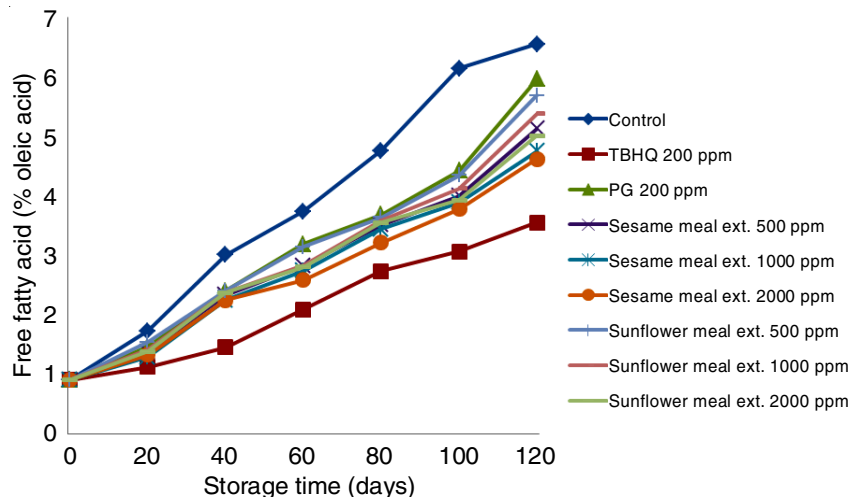

Fig. 3. Comparison of free fatty acid (\% oleic acid) of crude soybean oil stored at $50{ }^{\circ} \mathrm{C}$

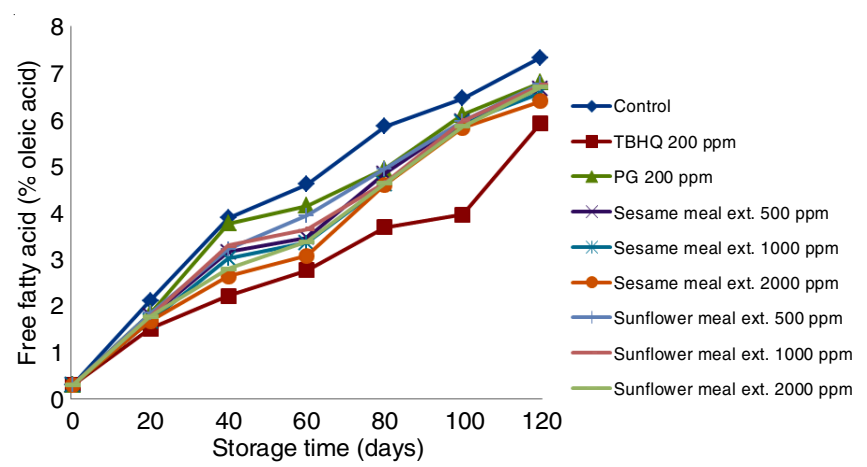

Fig. 4. Comparison of free fatty acid (\% oleic acid) of refined soybean oil stored at $50{ }^{\circ} \mathrm{C}$

the oil sample containing extracts slightly decreased as the concentration of extracts increased in the oil. Hence, increase in the extract concentration in the oil reduces the rate at which oil undergoes oxidative rancidity. The results demonstrated that sesame meal extract had higher antioxidant activity than sunflower meal extract and propyl gallate but lower activity than that of tert-butylated hydroxyquinone for all concentrations.

Effect on $p$-anisidine value: The $p$-anisidine value is based on the reactiveness of aldehyde carbonyl bond on $p$-anisidine amine group, leading to the formation of Schiff base that absorbs at $350 \mathrm{~nm}$ [20]. Fig. 5 depicted $p$-anisidine value for crude soybean oil samples stabilized with the extract, propyl gallate, tertbutylated hydroxyquinone and control. It was clearly observed that all the varying concentrations of sesame and sunflower extracts were effective in lowering the $p$-anisidine value of crude soybean oil than $200 \mathrm{ppm}$ propyl gallate. The capability of these extracts to reduce $p$-anisidine value of crude soybean oil slightly increased as the concentration of extract increased. It was also revealed sesame meal extract is more stable than sunflower meal extract. This is in accordance with Chatha et al. [21]. They found that supplementation of canola oil with wheat bran extract strongly inhibited the rise of anisidine value during the storage period. Fig. 6 presents the $p$-anisidine value of refined soybean oil stored with natural as well as synthetic antioxidants for 120 days. Throughout the 120 days of storage the $p$-anisidine value of refined soybean oil that contained no additive was higher than oil samples that contained additives (extracts, propyl gallate, tert-butylated hydroxyquinone). As the concentration of sesame and sunflower extracts increased in the oil sample, $p$-anisidine value of the oil decreased remarkably. 


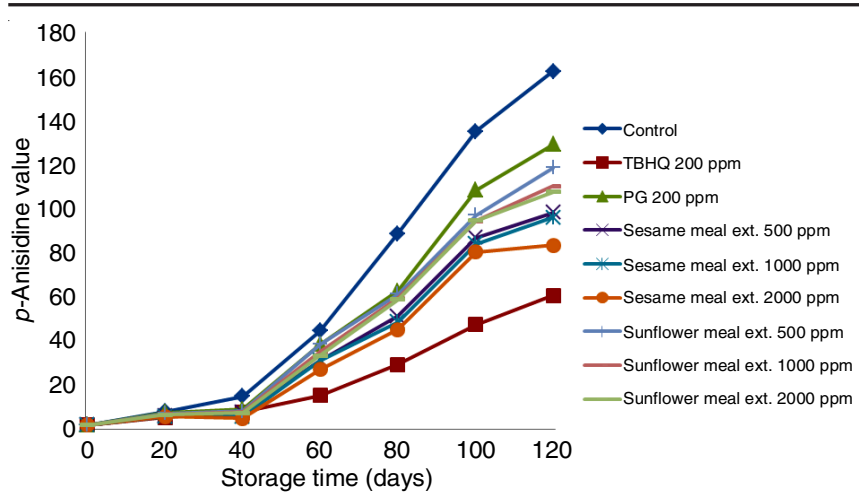

Fig. 5. Comparison of $p$-anisidine value of crude soybean oil stored at $50{ }^{\circ} \mathrm{C}$

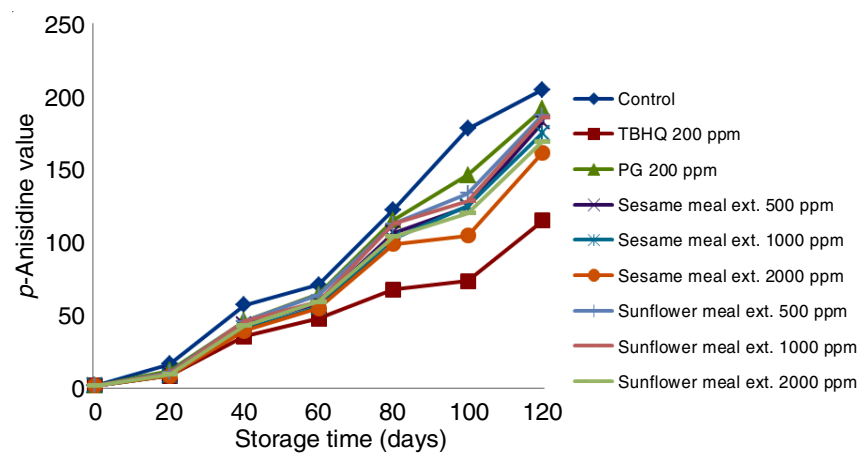

Fig. 6. Comparison of $p$-anisidine value of refined soybean oil stored at $50{ }^{\circ} \mathrm{C}$

During storage, sesame and sunflower extracts were more effective than 200 ppm propyl gallate while less than tertbutylated hydroxyquinone in reducing $p$-anisidine value of refined soybean oil. The result is in agreement with data reported by Aruoma et al. [22]. They reported that in soybean oil, carnosic acid was more active than butylated hydroxyquinone and butylated hydroxy anisole, but less active than tert-butylated hydroxyquinone. Difference in antioxidant activity of different antioxidants may be due to chemical structures. The stability of phenoxy radicals reduces the rate of propagation and further reactions and increases the oxidative stability of lipids [20].

\section{Conclusion}

It is concluded that both sesame and sunflower meal extracts can stabilize crude and refined soybean oils effectively stored in transparent glass container. The antioxidant activity of both extracts was higher than that of $200 \mathrm{ppm}$ propyl gallate but lower than $200 \mathrm{ppm}$ tert-butylated hydroxyquinone at $50{ }^{\circ} \mathrm{C}$. While sesame meal extract is more effective than sunflower meal extract in stabilization of crude and refined soybean oil. This shows that sesame and sunflower meal extracts extended the stability even more than the standard one. The result demons- trated that refined oil is more susceptible to oxidation as compare to crude oil because natural antioxidants such as total tocopherol and carotenoid had been lost during refining.

\section{ACKNOWLEDGEMENTS}

One of authors (Anjani) is grateful to University Grants Commission, New Delhi, India for awarding the Junior Research Fellowship.

\section{REFERENCES}

1. D. Krishnaiah, R. Sarbatly and R. Nithyanandam, Food Bioprod. Process., 89, 217 (2010); https://doi.org/10.1016/j.fbp.2010.04.008.

2. F. D. Gunstone and F. A. Norris, Lipid in Food: Chemistry, Biochemistry and Technology, Pergamon Press New York, p. 58 (1983).

3. P.P. Coppen, eds.: J.C. Allen and R.J. Hamilton, Rancidity in Foods, Elsevier Applied Science, London, UK, edn 2, p. 83 (1989).

4. M. Namiki, CRC Crit. Rev. Food Sci. Nutr., 29, 273 (1990); https://doi.org/10.1080/10408399009527528.

5. C. Pokorny, L. Trojakova, N. Yanishlieva, and M. Gordon, Antioxidants in Food, Woodhead Publishing Ltd. (England), p. 355 (2001).

6. Y.S. Shyu and L.S. Hwang, Food Res. Int., 35, 357 (2002); https://doi.org/10.1016/S0963-9969(01)00130-2.

7. M. Namiki, Food Rev. Int., 11, 281 (1995); https://doi.org/10.1080/87559129509541043.

8. A.A.A. Mohdaly, I. Smetanska, M.F. Ramadan, M.A. Sarhan and A. Mahmoud, Ind. Crops Prod., 34, 952 (2011);

https://doi.org/10.1016/j.indcrop.2011.02.018.

9. F. Shahidi, C.M. Liyana-Pathirana and D.S. Wall, Food Chem., 99, 478 (2006); https://doi.org/10.1016/j.foodchem.2005.08.009.

10. S.F. Hamed, M. Suzanne and M.G. Megahed, Life Sci. J., 9, 320 (2012).

11. F. Kreps, L. Vrbikova and S. Schmidt, Int. J. Eng. Res. Appl., 4, 45 (2014).

12. N. Wanjari and J. Waghmare, Adv. Appl. Sci. Res., 6, 221 (2015).

13. AOAC, Official Methods of Analysis, Association of Official Analytical Chemists: Washington, DC (1990).

14. American Oil Chemists' Society, Official Methods and Recommended Practices of the American Oil Chemists' Society, AOCS Press: Champaign, IL, edn 5, p. 18 (1998).

15. K.L. Nyam, M.M. Wong, K. Long and C.P. Tan, Int. Food Res. J., 20, 695 (2013).

16. M. El-Mallah and S. El-Shami, Agric. Biol. J. North Am., 2, 341 (2011); https://doi.org/10.5251/abjna.2011.2.2.341.349.

17. F.M.A. Rehab, E-J. Environ. Agric. Food Chem., 9, 396 (2010).

18. J.O. Arawande and I.A. Amoo, Pak. J. Sci. Ind. Res., 52, 303 (2009).

19. J.O. Arawande, I.A. Amoo and L. Lajide, Bangladesh J. Sci. Ind. Res., 49, 249 (2014); https://doi.org/10.3329/bjsir.v49i4.22628.

20. Y. Zhang, L. Yang, Y. Zu, X. Chen, F. Wang and F. Liu, Food Chem., 118, 656 (2010); https://doi.org/10.1016/j.foodchem.2009.05.038.

21. S.A.S. Chatha, A.I. Hussain, J.R. Bajwa and M. Sagir, J. Food Lipids, 13, 424 (2006); https://doi.org/10.1111/j.1745-4522.2006.00068.x.

22. O.I. Aruoma, B. Halliwell, R. Aeschbach and J. Löligers, Xenobiotica, 22, 257 (1992); https://doi.org/10.3109/00498259209046624. 